\title{
Chemically Stable High Resolution Surface Patterning by Thiolated DNA for Self Assembly of Nanocircuits on a Gold Nano-Dot Surface
}

\author{
Nitesh Madaan, ${ }^{1}$ Aaron Terry, ${ }^{2}$ Robert C. Davis, ${ }^{3}$ Helmut Schlaad, ${ }^{4}$ Matthew R. Linford ${ }^{1}$ \\ ${ }^{1}$ Department of Chemistry and Biochemistry, Brigham Young University, Provo UT 84602 \\ ${ }^{2}$ Department of Chemical Engineering, Brigham Young University, Provo UT 84602 \\ ${ }^{3}$ Department Physics and Astronomy, Brigham Young University, Provo UT 84602 \\ ${ }^{4}$ Max Planck Institute of Colloids and Interfaces, MPI KGF Golm 14424 D-Potsdam, Germany
}

Someday, nanocircuits may perform complex calculations at high speeds using low power. Indeed, the need and constraints regarding complex interconnections in such nanometer scale circuits suggests that self-assembly will be a comparatively easier approach than relying solely on a top down strategy. DNA templated segments (circuit elements) of a large circuit made with DNA origami ${ }^{1}$ and high resolution patterning of the surface by complementary DNA templates may also assist in the self assembly of nanocircuits. Semiconducting elements (carbon nanotubes) might be introduced into circuit elements to act as transistors. The self-assembled nanocircuit can be selectively metalized to make the circuit conducting. Previously, thiols have been patterned onto a gold surface by dip-pen nanolithography. ${ }^{2}$ However, although the Au-S bond is initially fairly strong, once sulfur oxidizes this bond becomes weak. An alternative approach, which we propose, is to self assemble a dithiol monolayer onto a gold nano-dot surface followed by reaction with 1,4-polybutadiene (thiol-ene chemistry). ${ }^{3}$ Although, the dithiol monolayer should still be prone to oxidation, there will now be many of these weak Au-S (oxidized) bonds that should be cross-linked by 1,4-polybutadiene (PBd). This surface should also have many surface carbon-carbon double bonds that could be used to attach the thiolated complementary DNA required for surface patterning and immobilization of DNA origami. Such an assembly should make the entire structure more stable, reducing the likelihood that DNA templated circuit elements will detach. The 1,4-polybutadiene molecular cover should also slow the oxidation of sulfur by reducing the diffusion of oxygen to some extent. Along with thiolated complementary DNA, another thiolated molecule with a phosphate end group could be attached to PBd-terminated gold nanodots. The repulsive electrostatic interaction between phosphate end groups and the phosphate groups of DNA-templated circuit elements should assist in selective DNA binding. To date, the attachment of polybutadiene to a dithiol monolayer followed by attachment of another thiol moiety (perfluorodecanethiol) onto the surface double bonds has been successfully achieved on a flat $\mathrm{Au}(111)$ surface. The attachment of thiolated DNA to a $\mathrm{PBd} /$ mercapto silane-coated silicon wafer has also been achieved. Surfaces were characterized by contact angle goniometry, spectroscopic ellipsometry, time-of-flight mass spectrometry, and X-ray photoelectron spectroscopy. Scanning electron microscopy and atomic force microscopy will also be used for characterization of these new materials. In the near future, the stability profile of these polymer-modified surfaces, compared to thiols attached directly to a gold, surface will be studied. We will also demonstrate attachment of thiolated complementary DNA to surface double bonds on planar surfaces, followed by attachment of DNA-templated circuit elements. Once we achieve these goals, the same chemical modification strategy will be applied to gold nano-dot surfaces. 


\section{References}

1. Pound, E.; Ashton, J. R.; Becerril, H. A.; Woolley, A. T., Nano Lett. 2009, 9 (12), 4302-4305.

2. Sheehan, P. E.; Whitman, L. J., Phys. Rev. Lett. 2002, 88 (15), 156104.

3. Wickard, T. D.; Nelsen, E.; Madaan, N.; Brummelhuis, N. T.; Diehl, C.; Schlaad, H.; Davis, R. C.; Linford, M. R., Langmuir 2010, 26 (3), 1923-1928.

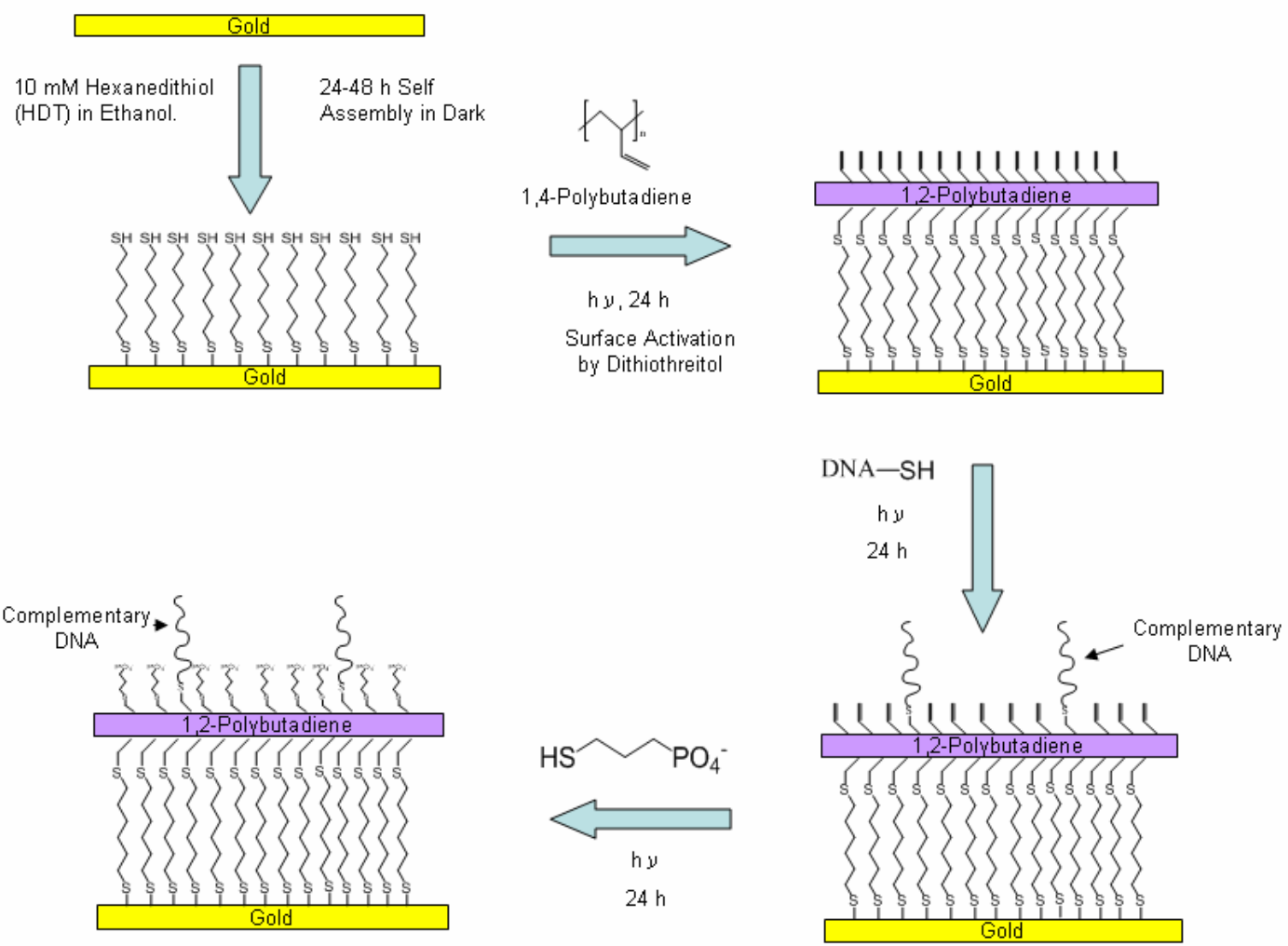

Chemical Modification Scheme

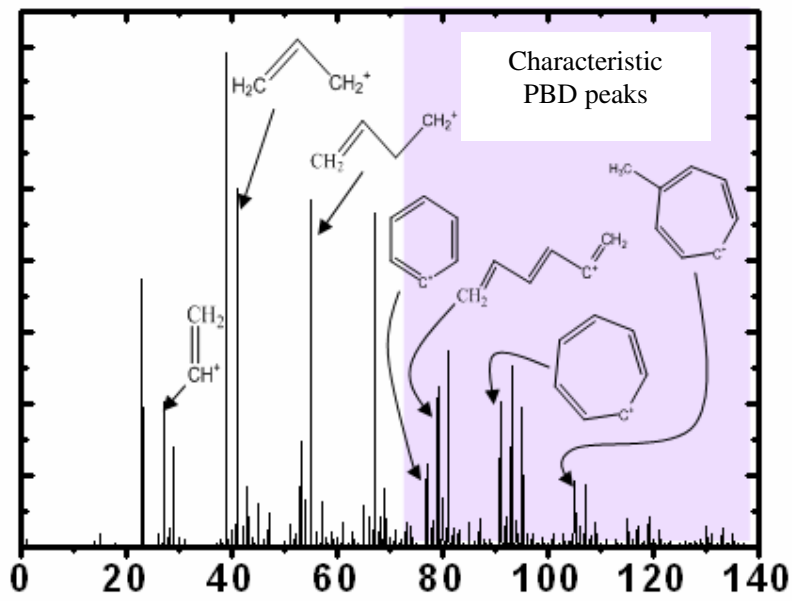

Positive Ion Mode ToF-SIMS spectrum of Polybutadiene/Hexanedithiol/Gold Surface 